\title{
Normas de transición y pensión de vejez en Colombia. Un análisis del sistema normativo desde la teoría del derecho*
}

\author{
Transitional Rules and Retirement Pension \\ in Colombia. An Analysis of the Legal System \\ from a Legal Theory Perspective
}

Normas de transição e aposentadoria por idade na Colombia. Uma análise do sistema normativo a partir da teoria do direito

Andrés Raúl Camargo Rodríguez ${ }^{* *}$
* El presente trabajo es el resultado de una investigación adelantada dentro del marco del máster avanzado en Ciencias Jurídicas de la Universitat Pompeu Fabra. Fue elabo- rado bajo la orientación de Josep María Vilajosana, a quien expreso toda mi gratitud.
** https://orcid.org/0000-0002-0748-6686. Universidad Pompeu Fabra, España. andresraul.camargo01@estudiant.upf.edu

Recibido: 27/08/2019. Envío a pares: 16/03/2020 Aprobado por pares: 29/03/2020. Aceptado: 02/05/2020

DOI: 10.5294/dika.2020.29.1.1 


\section{Resumen}

En Colombia, en el contexto de la seguridad social, existe el derecho al reconocimiento de una prestación económica que reemplaza el ingreso en la etapa de vejez -pensión de vejez-. La norma que contempla el derecho ha sido objeto de modificaciones en las últimas décadas. En ese contexto surgen las "normas de transición", variedad específica de reglas jurídicas que, prima facie, permiten la aplicación de normas no vigentes al momento en que se declara la existencia de ese derecho por cuenta del sujeto que tiene asignada dicha función. Así, el objeto de este trabajo consiste en explicar este fenómeno a través de los conceptos que provee la teoría del derecho en una versión positivista-analítica. Para el efecto, la metodología escogida supone: i) la reconstrucción del concepto de sistema jurídico -en cuanto explicandum-, junto con algunos de los elementos que lo conforman; ii) la descripción de los sistemas normativos que han regulado la pensión de vejez en Colombia desde su aparición en la legislación; iii) la ubicación del problema de las normas de transición dentro de la estructura conceptual pertinente al sistema jurídico; iv) la identificación de algunos aspectos controvertidos de la teoría a partir del caso descrito. Las conclusiones pretenden establecer un diálogo recíproco entre la teoría del derecho y la dogmática jurídica con el fin de proveer una visión alternativa del fenómeno.

\section{Palabras clave}

Aplicabilidad; aplicabilidad externa; aplicabilidad interna; criterios de aplicabilidad; derogación; dinámica; norma de transición; orden estatal; orden jurídico; pensión de vejez; pertenencia; tiempo; validez. 


\section{Abstract}

In Colombia, the set of rules regulating retirement pensions has been subject to a wide array of amendments during the last decades. In that context "normas de transición" -a specific variety of legal rules which allow applying other "not in force rules" came into existence. The purpose of this article is to explain the phenomenon by using concepts provided by an analytic-positivistic version of legal theory. Hence, the methodological approach pursues: i) Reconstructing the concept of the legal system -as explicandum- and some of the key elements that make it up, ii) Describing retirement pension Colombian legal system from the outset iii) Locating the "normas de transición" within the categories related to the concept of the legal system; and iv) Underlining some of the controversial issues in the subject from the case. The conclusions are addressed at setting a dialogue between legal theory and specialized legal studies to present an alternative approach to the matter.

\section{Keywords}

Applicability; external applicability; internal applicability; applicability criteria; derogation; legal system; dynamic; normas de transición; legal order; retirement pension; time; validity. 


\section{Resumo}

Nas últimas décadas o conjunto de normas relativas à aposentadoria por idade foi objeto de múltiplas modificações nas distintas na Colômbia. Nesse contexto, as denominadas "normas de transição" - tidas como uma subespécie de norma jurídica que, prima facie, permite a aplicação de normas não-vigentes foram implementadas. Destarte, o objetivo deste trabalho consiste em explicar tal fenômeno por meio de conceitos fornecidos pela teoria do direito em sua vertente positivista-analítica. Para tanto, a metodologia escolhida contempla: (i) a reconstrução do conceito de sistema jurídico - enquanto explicandum em conjunto com alguns de seus elementos constitutivos; (ii) a descrição dos sistemas normativos que - na experiência colombiana - regularam a aposentadoria por idade desde a sua origem; (iii) a alocação do problema das normas de transição em alguma das categorias relacionadas com o conceito de sistema jurídico; e, por fim, (iv) a identificação de alguns aspectos controvertidos da teoria a partir da descrição de um caso. Em suma, as conclusões do estudo pretendem estabelecer um diálogo recíproco entre a teoria do direito e a dogmática jurídica, para que - assim - seja ofertada uma visão alternativa ao fenômeno.

\section{Palavras-chave}

Dinâmica. Sistema jurídico; Ordem jurídica; Ordem Estatal; Norma de transição; Validez; Tempo; Adesão; A plicabilidade; Aposentadoria por idade; Derrogação; Regime de Transição; Regra de identificação. 
Sumario. I. Marco conceptual: sistema jurídico y otros conceptos relevantes. 1. Orden estatal, orden jurídico y sistema jurídico. 2. El concepto de tiempo y los sistemas jurídicos. 3. Validez como pertenencia y aplicabilidad. 3.1. Pertenencia. Criterios. 3.2. Aplicabilidad. 3.2.1. Aplicabilidad externa. 3.2.2. Aplicabilidad interna. 3.2.3. Relación entre aplicabilidad interna y aplicabilidad externa. 3.2.4. Normas aplicables no pertenecientes. 3.2.5 Normas pertenecientes no aplicables. II. Dinámica de las normas que regulan la pensión de vejez en Colombia. 1. Primer orden jurídico de referencia: Constitución Política de 1886. 2. Orden jurídico actual: Constitución Política de 1991. III. Casos problemáticos. 1. Normas de transición: ambigüedad. 2. Indeterminación semántica. 3. Jerarquías y conflictos. IV. Conclusiones. Bibliografía

\section{Marco conceptual: sistema jurídico y otros conceptos relevantes}

\section{Orden estatal, orden jurídico y sistema jurídico}

El concepto de sistema jurídico ha ocupado la atención de las teorías del derecho de corte positivista, a partir del estudio de las relaciones existentes entre las normas jurídicas. En ese marco, la teoría provee criterios útiles para identificar el material jurídico que regula la vida colectiva durante un momento determinado.

La expresión sistema jurídico ha sido entendida en diferentes sentidos y, para el efecto, los teóricos han realizado distinciones conceptuales con el fin de recoger los diversos significados comúnmente atribuidos. Autores como $\operatorname{Raz}^{1} \mathrm{y}$ Bulygin $^{2}$ (1991) dieron los primeros pasos en esta dirección al distinguir entre sistemas y órdenes, y otros han planteado la necesidad de efectuar un análisis en niveles en los que además se incluyan elementos superiores como el orden internacional y el orden estatal. ${ }^{3}$

Así, en primer lugar aparecen nociones como la de orden estatal, que da cuenta de los límites espaciales en los cuales el derecho se desarrolla sistemáticamente. Así, "el orden estatal ofrece el marco en el que se encuadra la sucesión de sistemas jurídicos, $\mathrm{y}$, al mismo tiempo, permite separar el orden jurídico vigente en el mismo momento pero en un territorio distinto". ${ }^{4}$ La necesidad de incluir este concepto en el estudio deriva de una acepción fundamental: una norma es aplicable a un espacio geográfico determinado y, por ello, distinguible de otra, que para ese mismo momento regula un caso determinado en un sitio diferente.

Joseph Raz, The concept of a legal system. An introduction to the theory of a Legal System, Oxford, Clarendon, 1980. Eugenio Bulygin, "Time and Validity", en Análisis lógico y derecho, Centro de Estudios Constitucionales, Madrid, 1991, pp. 195-215.

3 Aun cuando el autor incluye un nivel superior denominado orden internacional, él mismo señala que este no es necesario tratarlo, o al menos que ello carece de relevancia en determinados supuestos (Josep M. Vilajosana, “Dinámica de sistemas y persistencia de normas jurídicas", Doxa 21 [I] [1998], p. 342). Josep M. Vilajosana, El significado político del Derecho, México, Marcial Pons, 1997, p. 335. 
En un segundo nivel ${ }^{5}$ aparece la noción de orden jurídico, respecto de la cual Alchourrón y Bulygin ${ }^{6}$ plantearon ocho tesis que recogen el significado comúnmente atribuido por los juristas. Tales postulados incluyen acepciones relativas a este como la definición incompleta de un concepto (en cuanto refiere la totalidad de normas validas de acuerdo con un criterio específico), como conjunto de normas, como sistema normativo, además de algunas características distintivas, como su carácter dinámico, su persistencia (planteada en términos de identidad), la determinación o determinabilidad de su contenido y la unicidad de este dentro del Estado.

Sin ahondar en particularidades de cada acepción, y sobre todo en las dinámicas de interacción entre unas y otras, es necesario resaltar la dificultad que existe para conciliar la tesis según la cual el orden jurídico es un conjunto de normas con aquella que se refiere a su identidad. Así, asumir que el orden jurídico es un conjunto implica aceptar que este cambia por otro conjunto cuando se da la sustitución o eliminación de alguno de sus elementos; mientras tanto, la idea de identidad sugiere que este "puede perdurar en el tiempo [...], aun cuando su contenido sea diferente en cada momento". ${ }^{7}$

Una de las principales aportaciones dirigidas a superar la dificultad teórica es atribuible a Joseph Raz, quien por primera vez introdujo la noción de temporalidad en el análisis del derecho como sistema. ${ }^{8}$ Para ello, Raz distinguió entre sistemas jurídicos "continuos o no momentáneos" y "sistemas jurídicos momentáneos". ${ }^{\prime 9}$

Existen diferentes críticas a los planteamientos de Raz. ${ }^{10}$ Una de las principales objeciones proviene de Bulygin, quien explica la ambigüedad como una cuestión referida a la forma en que se produce la relación entre sistemas continuos y entre sistemas momentáneos

... no es claro si "sistema jurídico" Raz entiende un conjunto de sistemas momentáneos o un conjunto de normas, a saber, todas las normas que pertenecen a todos

5 La explicación de las dinámicas de interacción entre normas a través de "niveles" es introducida por Vilajosana, ibid.

Carlos Alchourrón y Eugenio Bulygin, Normative Systems, Springer, Wien, 1976, p. 5.

Idem.

Véase Alchourrón y Bulygin, Normative Systems, op. cit.; Ricardo Caracciolo, El sistema jurídico: problemas actuales, Madrid, Centro de Estudios Constitucionales, 1988; Bulygin, "Time and Validity", op. cit.; José Juan Moreso y Pablo Navarro, Orden jurídico y sistema jurídico. Una investigación sobre la identidad y dinámica de los sistemas jurídicos, Madrid, Centro de Estudios Constitucionales, 1993; Vilajosana, El significado político del Derecho, op. cit.

9 Raz, The concept of a legal system, op. cit.

10 Por ejemplo, Bulygin sugiere que la caracterización de los sistemas como entidades "momentáneas" se presta a confusión, dado que estos son de corta duración cuando en realidad la duración es relativa a un intervalo temporal entre actos de creación y derogación (Eugenio Bulygin, "Time and Validity", en Análisis lógico y derecho, Centro de Estudios Constitucionales, Madrid, 1991, p. 259); Moresso y Navarro cuestionan la imposibilidad de identificar sistemas jurídicos vacíos (Moreso y Navarro, Orden jurídico y sistema jurídico, op. cit., p. 44), y Vilajosana sostiene que la noción de sistema no momentáneo o continuo coincide en algunos apartes con aquella de orden jurídico, y en otros con un nivel superior que denomina orden estatal (Vilajosana, El significado político del Derecho, op. cit., p. 21). 
los sistemas momentáneos que corresponden al sistema jurídico en cuestión. Si el sistema jurídico es un sistema momentáneo, la relación entre un sistema momentáneo y el sistema jurídico es la de pertenencia; si es un conjunto de normas y los sistemas momentáneos son subclases del sistema jurídico, entonces la relación es la de inclusión. La terminología de Raz es vacilante en este punto. (p. 260)

Como explican Moresso y Navarro, la existencia de una relación de inclusión implica que los sistemas momentáneos son subconjuntos del sistema continuo (el cual a su vez debe ser entendido como un conjunto) durante un momento determinado $t$; mientras que en el caso de la relación de pertenencia, el sistema no momentáneo es una secuencia de sistemas, cada uno de los cuales hace parte de dicha entidad continua debido a que aquel detenta una propiedad específica. Así: i) los órdenes están conformados por sistemas, no por normas; y ii) los sistemas normativos no pueden ser vacíos, pues "es lógicamente imposible identificar entre dos sistemas momentáneos $\mathrm{Sj}$ y Sj' un segmento temporal t que no contenga ningún sistema momentáneo, y simultáneamente, afirmar que $\mathrm{Sj}$ y Sj' pertenecen al mismo orden jurídico". ${ }^{11}$

Sobre la base de este aparato conceptual, y con el fin de eliminar la ambigüedad de la que se acusa a Raz por el uso indeterminado del lenguaje, Alchourrón y Bulygin ${ }^{12}$ propusieron una nueva distinción entre sistema y orden jurídico. En virtud de esta, asignaron a la primera convención el carácter de conjunto de normas (criterio de inclusión), mientras que respecto al "orden" fue considerado como secuencia de sistemas jurídicos (criterio de pertenencia).

\section{El concepto de tiempo y los sistemas jurídicos}

La diferenciación de Raz entre sistemas jurídicos momentáneos y no momentáneos permitió identificar la importancia del tiempo como un componente del sistema jurídico. De otro lado, la tesis de Alchourrón y Bulygin según la cual cada sistema es un conjunto implica que la adición o eliminación de elementos genera la sustitución por nuevas entidades.

En relación con el primer componente, Bulygin ${ }^{13}$ contribuyó mediante la elaboración de categorías posteriores, en virtud de las cuales se propone distinguir: el tiempo externo del sistema, el tiempo externo de la norma y el tiempo interno de la norma. La primera noción corresponde al intervalo existente entre los actos de introducción y derogación dentro de los cuales se predica la pertenencia de una norma jurídica a un sistema; la segunda con referencia a los mismos lími- 
tes en un espacio temporal más amplio, es decir, cuando la norma pertenece a varios sistemas del mismo o de diferentes órdenes. Finalmente, el concepto de tiempo interno hace referencia, ya no a un ámbito de pertenencia, sino de aplicabilidad, esto es, a todos los momentos en los que la norma puede ser utilizada para resolver casos concretos independientemente de la satisfacción de los criterios de pertenencia.

Ahora, en ciertas relaciones dinámicas, los tiempos interno y externo no corresponden al mismo intervalo (e. g., el primero es más amplio que el segundo debido a la extensión de los efectos normativos más allá del ámbito de derogación, o en el caso de inaplicación de una norma durante un espacio temporal determinado dentro de otro momento más amplio).

\section{Validez como pertenencia y aplicabilidad}

Un sistema jurídico, como estructura básica (perteneciente a un orden jurídico Oj, en un orden estatal Oe), es un conjunto no vacío, cuyas normas son aplicables durante un momento determinado $t$, que varía como consecuencia de actos materiales de promulgación o de derogación.

Intuitivamente, se puede pensar que todos los casos particulares que ocurren durante el momento determinado $t$ son gobernados por las normas pertenecientes a dicho conjunto. Sin embargo, las dinámicas normativas proponen ciertos desafíos. En diversas ocasiones, los agentes estatales con funciones de aplicación (jueces, autoridades administrativas, particulares, inter alia) deben recurrir a normas que ya no pertenecen ${ }^{14}$ a los sistemas jurídicos, o viceversa, las normas que pertenecen a los sistemas jurídicos no pueden regular la totalidad de los casos que se producen durante el tiempo de los sistemas.

Así, una norma es válida en tanto pertenece a un sistema jurídico, y en la medida que debe ser aplicada para resolver un caso determinado. ${ }^{15}$ En ese contexto aparecen las nociones de pertenencia y aplicabilidad como distintivas de validez.

\subsection{Pertenencia. Criterios}

Aceptar una representación conceptual del orden jurídico en niveles (sistema y orden) crea una serie de problemas en términos de identidad y de estructura. ${ }^{16}$ A través de la noción de identificación se plantea la cuestión de la pertenencia de las normas a los sistemas, y de los sistemas a los órdenes.

14 Supra, numerales 3.1. y 3.2.

15 Bulygin, "Algunas consideraciones sobre los sistemas jurídicos", op. cit.

16 Caracciolo, El sistema jurídico: problemas actuales, op. cit., p. 28. 
En diferentes trabajos Bulygin ${ }^{17}$ ha planteado una regla de identificación (RI), con el propósito de proveer una respuesta conceptual útil a fin de delimitar en un número finito de pasos el contenido de los sistemas y de los órdenes. Esta regla ha sido expresada en el siguiente esquema:

1. El conjunto de normas independientes ( $\mathrm{Ni} 1, \mathrm{Ni} 2, \ldots, \mathrm{Nin})$ es el sistema originario del orden jurídico $\mathrm{Oj}$.

2. Si una norma de competencia Nc, válida en el sistema S1 $(t)$, que pertenece al orden $\mathrm{Oj}$, autoriza a la autoridad A a promulgar la norma $\mathrm{N}$ y A promulga $\mathrm{N}$ en el tiempo $t$, entonces $\mathrm{N}$ es válida en el sistema S2 ( $\mathrm{t}+1)$ de Oj (correspondiente al momento siguiente a $t$ ).

3. Si una norma de competencia Nc, válida en el sistema S1 ( $t)$, que pertenece al orden $\mathrm{Oj}$, autoriza a la autoridad $\mathrm{A}$ a derogar la norma $\mathrm{N}$, que es válida en $\mathrm{S} 1$ $(t)$, y A deroga $\mathrm{N}$ en el tiempo $t$, entonces $\mathrm{N}$ no es válida en el sistema S2 ( $t+1)$ de $\mathrm{Oj}$ (correspondiente al momento siguiente a $t$ ).

4. Las normas válidas en el sistema S1 $(t)$, que pertenecen al orden $\mathrm{Oj}$, que no han sido derogadas en el tiempo $t$, son válidas en el sistema S2 $(\mathrm{t}+1)$ de Oj (correspondiente al momento siguiente a $t$ ).

5. Todas las consecuencias lógicas de las normas válidas del sistema S1 (t), que pertenece al orden $\mathrm{Oj}$, también son válidas en $\mathrm{S} 1(t) .{ }^{18}$

La regla número 1 introduce la noción de norma independiente, útil para definir su equivalente contradictorio: las normas dependientes. Así, una norma independiente es aquella cuya pertenencia a un sistema jurídico no está condicionada por la pertenencia previa de otras normas al sistema, sino por "conceptos extrasistemáticos que constituyen el punto de partida de las relaciones normativas propias" ${ }^{19}$ Dichas normas, en cuanto pertenecientes, se diferencian en la medida que no provienen de relaciones genéticas de legalidad o deducibilidad. Los sistemas constituidos por estas normas generalmente son denominados originarios (o primera Constitución), y la cuestión relativa a la pertenencia al primer orden simplemente no se plantea, puesto que la secuencia de derivación dinámica comienza con ella: se trata, por definición, del primer eslabón de esa cadena. ${ }^{20}$ Por su parte, las normas dependientes son aquellas cuya pertenencia al sistema está condicionada por la pertenencia de otras normas. ${ }^{21}$

Con respecto a tal variedad, los teóricos han trazado una distinción a partir del origen. Así, existen vínculos genéticos de dependencia por razones de le-

17 Alchourrón y Bulygin "Sobre el concepto de orden jurídico", op. cit.; Bulygin, "Algunas consideraciones sobre los sistemas jurídicos", op. cit.

18 Eugenio Bulygin y Daniel Mendonça, Normas y sistemas normativos, Madrid, Marcial Pons, 2005, p. 50.

19 Caracciolo, El sistema jurídico: problemas actuales, op. cit., p. 31.

20 Bulygin y Mendonça, Normas y sistemas normativos, op. cit., p. 49.

21 Moreso y Navarro, Orden jurídico y sistema jurídico, op. cit., p. 37. 
galidad, u otros de carácter lógico denominado deducibilidad. ${ }^{22}$ Los enunciados $2^{\circ}$ y $3^{\circ}$ dan cuenta del vínculo que se basa en la legalidad, mientras que el $5^{\circ}$ se relaciona con aquel que surge por efectos de deducibilidad. En estos términos, la legalidad se entiende relacionada con la pertenencia (en tanto promulgación) o la no pertenencia (derogación o cualquier otra forma de exclusión), mientras que la deducibilidad solamente se reputa referida al ámbito de la pertenencia.

La noción de sistema como conjunto resulta útil para comprender la relación de legalidad (reglas 2a y 3a). Así, las normas que comparten una propiedad específica constituyen un conjunto denominado sistema jurídico (Sj), que a su vez existe en un conjunto mayor denominado orden jurídico (Oj). Cada nuevo conjunto Sj existente en Oj surge como consecuencia de la eliminación o introducción de algún elemento dentro de la misma secuencia $(S j+1, S j+2, \ldots$. $\mathrm{Sj}+\mathrm{t})$. Asimismo, cada sistema $\mathrm{Sj}$ tiene un momento específico $(t, t+1, t+2, \ldots t+n)$.

Un nuevo sistema pertenece al mismo orden cuando los cambios que dan origen a su creación se encuentran autorizados por una regla de competencia. ${ }^{23}$ Por el contrario, si el cambio se produce sin sujeción a una regla del sistema, el mismo puede constituir un cambio de orden (e. g. el caso de una revolución en la que se crea un nuevo sistema de normas independiente). Ahora, cuando la adopción o la exclusión normativa es exclusivamente formal, esto es, cuando se dictan nuevas normas que reiteran el contenido de otras existentes, o se derogan ya eliminadas, el cambio no produce el efecto de modificar el conjunto. ${ }^{24}$

En el modelo de deducibilidad la pertenencia de una norma $\mathrm{N}$ se basa, no en la existencia de otra norma que autoriza su expedición, ni en la ejecución de una acción determinada por una autoridad definida, sino en la existencia de otra norma en el sistema de la cual se deduce lógicamente.

En síntesis, la pertenencia de las normas a los sistemas jurídicos se funda en criterios de legalidad o deducibilidad, excepto en el caso de las normas independientes, respecto de las cuales no se exige ese vínculo genético.

\subsection{Aplicabilidad}

La idea de aplicabilidad se relaciona, en términos generales, i) con la producción de efectos jurídicos de las normas que hacen parte de los sistemas jurídi-

22 Caracciolo, El sistema jurídico: problemas actuales, op. cit., p. 57

23 Con respecto a este tipo de normas y a su posición dentro de la teoría general del derecho, véase Jordi Ferrer, Las normas de competencia: un aspecto de la dinámica jurídica, Madrid, Centro de Estudios Políticos y Constitucionales, 2000. 
cos; ii) con su carácter obligatorio; o iii) con el deber de hacer uso de ellas para resolver casos concretos por parte de aquellos sujetos que tienen a su cargo la función de adjudicación.

A partir del reconocimiento de múltiples acepciones de la aplicabilidad dentro del universo jurídico, y de algunas distinciones conceptuales, aquella puede ser abordada desde una perspectiva externa e interna.

\subsubsection{Aplicabilidad externa}

Así, la aplicabilidad externa tiene relación con la existencia de una norma $\mathrm{Nj}$ en un sistema $S$, que autoriza la aplicación de otra norma $\mathrm{N}$ en la resolución de un caso determinado c, que es la instancia de un caso general C. Como señalan Moreso y Navarro:

Una norma [N1] es externamente aplicable en un momento $t$, a cierto caso individual c que es una instancia de un caso genérico $\mathrm{C}$, si y solo si una norma $\mathrm{Nj}$, perteneciente a un sistema jurídico [S1] en $t$, prescribe (obliga o autoriza) la aplicación de N1 a los casos individuales que son instancias de C. ${ }^{25}$

Dicha noción se plantea, entonces, como una cuestión referida a la relación entre dos normas: una perteneciente (carácter derivado de la verificación de los requisitos que establece la RI) que prescribe el deber de aplicar otra norma determinada, y otra, perteneciente o no, que concretamente sirve a efectos de solucionar disputas. ${ }^{26}$

Como se puede constatar, el escenario planteado facilita la aproximación hacia el fenómeno de interacción entre las dos funciones constitutivas de validez: pertenencia y aplicabilidad. Moreso y Navarro señalan que una norma (perteneciente o no), puede ser aplicada, si y solo si, otra norma (que debe ser perteneciente), autoriza su aplicación. Bajo este planteamiento, entonces, es posible admitir la existencia de normas aplicables que no son pertenecientes. A este respecto, Bulygin apunta a la existencia de criterios de origen constitucional, legal, jurisprudencial, e incluso dogmático, que "tienen la forma de directiva dada a los jueces que establecen qué normas deben aplicarse a un caso dado". ${ }^{27}$

Algunos teóricos dirigen críticas en contra del planteamiento de los criterios de aplicabilidad de Bulygin. Por ejemplo, para Mendonça, según la interpre- 
tación de Rodríguez ${ }^{28}$ existe la necesidad de distinguir entre la obligación de aplicar ciertas normas para solucionar una controversia determinada, de la tarea de identificar concretamente el material normativo que se debe utilizar para resolver, como consecuencia de la satisfacción de ciertos requisitos. El primer caso mencionado hace referencia a los criterios de aplicabilidad desde una perspectiva normativa, mientras en el segundo caso se habla de dichos criterios desde una perspectiva conceptual. Tal distinción genera diferencias fundamentales, pues el desconocimiento de una regla de conducta crea un juicio de reproche asociado a la idea de desobediencia, mientras que el desconocimiento de la regla conceptual puede ser atribuible a la ignorancia u a otros factores.

En todo caso, la crítica que se dirige a Bulygin consiste en no efectuar una distinción clara entre estas dos dimensiones de la aplicabilidad (conceptual y normativa).

\subsubsection{Aplicabilidad interna}

El concepto de aplicabilidad interna hace referencia a las esferas de validez de las normas legales, en virtud de las cuales se restringe el ámbito de aplicación a ciertos casos específicos. Así, algunas normas que regulan un caso genérico limitan la producción de sus efectos a algún tiempo en específico, a un lugar, a algún grupo de individuos, o a alguna acción genérica. Por ello, los autores de esta distinción expresan esta forma de aplicabilidad en la siguiente definición: "Una norma es internamente aplicable en un momento $t$, a un caso individual $c$, si y solo si $c$ es una instancia de un caso genérico $C$, y $C$ es definido por las esferas de validez espacial, material, personal y temporal de $\mathrm{Ni}^{\prime \prime}{ }^{29}$

El caso de las normas que se aplican por virtud de la situación de transición entre sistemas normativos permite ilustrar con claridad la dimensión interna de la aplicabilidad. Como se verá, el legislador colombiano, en una práctica constante dentro de sistemas jurídicos pertenecientes al orden anterior, reconocía prestaciones de vejez distintas, según el grupo al que perteneciera cada individuo. Así, legalmente se establecía el derecho a recibir una suma de dinero periódica en la etapa de retiro por parte de los empleados públicos, trabajadores de las empresas privadas, o para aquellos sujetos que efectuaban contribuciones a un fondo de naturaleza común. Como las normas eran dirigidas a ciertos sujetos, y la propiedad se refería a la clase de acciones ejecutadas por dichos individuos, esas acciones se relacionan con el ámbito personal de validez.

28 Jorge Luis Rodríguez, Lógica de los sistemas jurídicos, Madrid, Centro de Estudios Políticos y Constitucionales,

29 Moreso y Navarro, "Applicability and effectiveness of legal Norms", op. cit., p. 206. 


\subsubsection{Relación entre aplicabilidad interna y aplicabilidad externa}

De la interacción entre los conceptos tratados se siguen algunos efectos que justifican su distinción. Tal es el caso de la inmutabilidad de la aplicabilidad interna por efectos de la aplicabilidad externa, o la existencia de una relación conceptual (condición necesaria) entre ellas.

\section{Como señalan Moreso y Navarro:}

Aún si la aplicabilidad interna de una norma Ni puede ser restringida o modificada por otra norma Nk, la aplicabilidad interna no puede ser descartada en favor de la aplicabilidad externa, porque la aplicabilidad externa de una norma Ni siempre requiere la aplicabilidad interna de otra norma $\mathrm{Nk}$, que pertenece a un sistema jurídico $\mathrm{S}$ y prescribe aplicar $\mathrm{Ni}^{30}$

Un ejemplo permite verificar la instrumentalidad del planteamiento: una norma Ni define un ámbito de validez personal (e. g. los empleados públicos tienen derecho al reconocimiento de una prestación de vejez), pero desaparece por efectos de la derogación a través de una norma Nk. Sin embargo, esa misma norma Nk autoriza la aplicación de la disposición derogada Ni, pero solo respecto de algunos casos en los que se cumpla una condición determinada (C) (e. g. tener una edad definida a la fecha de promulgación de Nk).

Aquí, Ni tiene un ámbito interno de aplicabilidad, determinado por una restricción en el carácter general de la norma, es decir, la aplicación a aquellos individuos que reúnen la condición de "empleado público" en el momento $(t)$ correspondiente al sistema en el cual perteneció. Así también, Nk constituye un ejemplo típico de criterio de aplicabilidad externo. Se trata de una norma que habilita la aplicación de otra norma (Ni), que dejó de ser perteneciente por efectos de la derogación. La función de $\mathrm{Nk}$ como criterio de aplicabilidad externo no obsta, sin embargo, para que Nk defina su propio ámbito de aplicabilidad interno; es decir, que restringa la aplicabilidad de $\mathrm{Ni}$ para un supuesto específicamente determinado (en este caso aquel descrito en forma de condición C). Aquí no se discute la pertenencia ni la aplicabilidad de $\mathrm{Nk}$, ni la simple aplicabilidad externa de Ni por efectos de Nk.

De esta manera, la aplicabilidad de Ni está determinada, de un lado, por su ámbito interno, y además por las condiciones que Nk establece. El ejemplo que se plantea-descartar la aplicabilidad interna de $\mathrm{Ni}$ en favor de la externa $\mathrm{Nk}$-implica aceptar que el derecho definido por Ni corresponde a todos aquellos sujetos que apenas cumplan con C. Ciertamente, el sentido de Nk es otro, y consiste en restringir la aplicabilidad interna de $\mathrm{Ni}$, al limitarla a aquellos

$30 \quad$ Ibid., p. 209. 
que, habiendo sido empleados públicos (ámbito de aplicabilidad interna de $\mathrm{Ni}$ ), cumplan con C (ámbito de aplicabilidad interno de $\mathrm{Nk}$ ).

Lo anterior lleva a plantear que la aplicabilidad interna se ocupa de la adecuación de un caso determinado $c$ a una norma $\mathrm{N}$ (¿puede una norma $\mathrm{N}$ regular un caso $c$ que es una instancia de un caso genérico $C$ ?), mientras que la dimensión externa hace referencia al deber u obligación del sujeto que aplica, de recurrir a una norma determinada (en este caso Nk) para solucionar la controversia puesta en su conocimiento. ${ }^{31}$

\subsubsection{Normas aplicables no pertenecientes}

La bibliografía especializada ha mostrado ejemplos que permiten ilustrar con mayor claridad supuestos en los que se produce la aplicabilidad sin pertenencia.

El primero de los casos es el de favorabilidad, incluido en materia penal en la mayoría de ordenamientos estatales modernos, y en virtud del cual se impone el uso de la norma más favorable para efectos de la imposición de una sanción jurídica asociada a una infracción legal. ${ }^{32}$

Existe un tercer supuesto relativo a la aplicación de normas extranjeras en el marco del derecho internacional privado, denominado reenvío, cuya existencia se verifica en ordenamientos como el español, y a través del cual se autoriza la aplicación de normas no pertenecientes al orden jurídico nacional. ${ }^{33}$

El último caso es el de la "prohibición de non liquet", también incluida en diversos ordenamientos procesales, según la cual, el juez no puede alegar la inexistencia de norma para abstenerse de dictar decisión frente a un caso concreto. En consecuencia, la existencia de una laguna normativa genera la obligación del operador judicial de recurrir a otras normas (incluso no pertenecientes al sistema) para resolver. Este supuesto se verifica en el caso del argumento $a$ contrario o del argumento analógico. ${ }^{34}$

En ese contexto, el caso de las normas de pensión de vejez del sistema colombiano, y en general cualquier otro sistema en otro orden que establezca un "régimen de transición" constituye un supuesto adicional a aquellos ya referenciados. Esto se debe a que, en este tipo de dinámica, las normas anteriores conservan el atributo de la aplicabilidad más allá de su derogación.

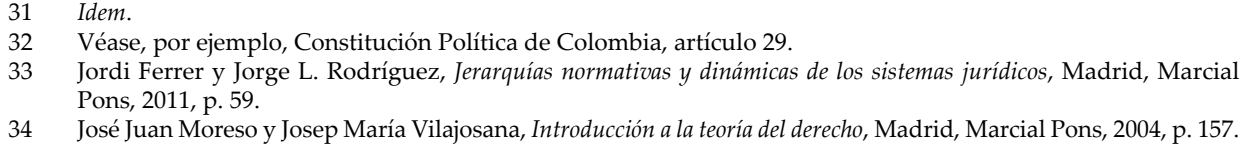


Al igual que en el mencionado caso de las normas extranjeras, dicha aplicación depende de la existencia de otras normas pertenecientes que obligan en este sentido a la autoridad, o en general a los individuos que tienen a su cargo la aplicación del derecho, a acudir a dichas normas para efectos de solucionar controversias concretas, además de la verificación de unos supuestos fácticos que ellas mismas definen. La ilustración del sistema colombiano da cuenta con mayor claridad del fenómeno.

\subsubsection{Normas pertenecientes no aplicables}

Ahora, existen casos de normas pertenecientes, conforme a los criterios tratados en el apartado 3.1., que no pueden ser utilizadas por los aplicadores para resolver determinados casos. Algunos ejemplos comunes dan cuenta de este fenómeno.

Es el caso de la no retroactividad o la favorabilidad penal. En el primer caso, la norma derogada extiende su aplicabilidad más allá del ámbito externo, debido a la creación de una restricción que impide al juez imponer sanciones establecidas en normas que no existían para la fecha en que se consumó un hecho con relevancia punitiva. Así, en dicho evento, a pesar de la existencia y aplicabilidad de una norma específica, el juez no puede acudir a ella para efectos de solucionar una disputa determinada.

Una lectura similar corresponde al caso de la favorabilidad, que ya ha servido para ilustrar la aplicabilidad sin pertenencia. Es el caso de una norma que fija una consecuencia más severa relacionada con la ejecución de una conducta determinada, la cual no se podría aplicar, pese a su pertenencia, para la resolución de situaciones determinadas debido a la existencia de una regla en el sistema que impone el deber de acudir a la norma más favorable.

Supuestos similares se presentan en el caso de la vacatio legis en el que, pese al acto de promulgación, la entrada en vigor o capacidad de la norma para regular un conjunto de casos, se difiere hasta una fecha determinada; o el de las antinomias, en las que el operador judicial o persona a quien se ha asignado la función de aplicar opta por no aplicar una norma perteneciente debido: i) a la existencia de un conflicto con otra norma; ii) la imposibilidad de aplicar simultáneamente ambas normas; y iii) la existencia de un criterio último que lo faculta para seleccionar una de las dos normas pertenecientes. ${ }^{35}$

Finalmente, se encuentra el caso de las normas pensionales, ya no de aquellas derogadas, sino de las que sustituyen a estas. En virtud de las normas de transición, las reglas que regulan derechos pensionales reducen su ámbito

$35 \quad$ Ibid., pp. 185-186. 
de aplicabilidad a los casos diferentes de aquellos para los que se autoriza la aplicación de normas anteriores. Así, si N establece que en el momento $t$ una pensión se causa a los 50 años de edad, y luego N1 sustituye a $\mathrm{N}$ y define que en el momento T1 la edad requerida para obtener tal prestación es de 55, y además hay una norma de transición NT que establece que para aquellas personas que a la fecha de promulgación de N1 cumplan con una condición determinada $\mathrm{C}$, entonces $\mathrm{N} 1$ no puede regular todos los casos que ocurren durante su tiempo externo, dado que NT autoriza el uso de N para solucionar ciertos casos.

\section{Dinámica de las normas que regulan la pensión de vejez en Colombia}

En los últimos apartados se desarrollaron algunas nociones útiles para la reconstrucción del concepto de sistema jurídico de acuerdo con la teoría del derecho de Alchourrón y Bulygin, y de los demás autores que han efectuado contribuciones en esta misma línea. Gracias a estos últimos han sido identificadas algunas de sus áreas problemáticas.

Esta sección está dedicada a mostrar las relaciones dinámicas del orden jurídico colombiano actual y su predecesor a través de los conjuntos conformados por normas que regulan los requisitos necesarios para obtener prestaciones de vejez o de jubilación. La constante sustitución de sistemas, la diferenciación entre tiempos internos y externos, la existencia de criterios de aplicabilidad, entre otros, permiten una visión más perspicua de las dinámicas y los conceptos hasta aquí tratados.

\section{Primer orden jurídico de referencia: la Constitución Política de 1886}

El punto de partida escogido es el orden jurídico inmediatamente anterior al actual, debido a que la mayor parte de normas aplicables y no pertenecientes provienen de conjuntos incluidos en este. Se trata de un orden jurídico distinto por una razón fundamental referida a la existencia de una modificación constitucional.

Dicho orden (en adelante Oj0) data del año 1886, cuando fue promulgada la versión original de la Constitución anterior. ${ }^{36}$ En Oj0 (esto es entre 1886 y 1991) surgió una secuencia de sistemas conformados por normas que regu-

36 La versión original de dicha Constitución fue sometida, según los especialistas, cuando menos a 65 modificaciones, de las cuales la dogmática se ha centrado en el análisis de aquellas correspondientes a los años 1910, 1936, 1945, 1957, 1968, 1975, 1977, 1979 y 1986. 
laron diferentes mecanismos de amparo de la población contra el riesgo de vejez (pensiones).

El primer sistema de esta secuencia, al que denominaremos S0, constituye un conjunto de normas independientes a través del cual se autorizó la actividad de producción normativa. Así, por ejemplo, al Congreso se le asignó la función de expedir normas reglamentarias (art. 120) y de crear empleos públicos junto con sus respectivas dotaciones (art. 76), mientras que al presidente le fueron asignadas tareas legislativas en determinadas circunstancias (art. 121).

A partir de allí se produjo la creación genética de los sistemas sucesivos, bien por simple derogación o sustitución, o bien por la adición de nuevos elementos.

El primero de esos sistemas (S1) surge por la introducción de un conjunto de normas que estableció, entre otras, el derecho a obtener pensiones de jubilación para trabajadores privados (N1) y públicos (N2) al completar 20 años de servicios y 50 años de edad. ${ }^{37}$

El surgimiento de S2 sobrevino como consecuencia de la sustitución de N1 por $\mathrm{N1}$ ', la cual estableció una edad superior para el reconocimiento del derecho a la pensión de jubilación de los trabajadores privados. ${ }^{38}$

El tercer sistema (S3) surgió en 1966, debido a la adición de normas que proveían medios específicos de protección contra la vejez de los trabajadores. ${ }^{39}$ Así, entonces, ocurrió el tránsito de un sistema en el que la obligación pensional estaba exclusivamente asignada a los empleadores (públicos y privados), a otro en el que, además, el Estado asumió parte de esa responsabilidad respecto de algunos beneficiarios, a través de un mecanismo denominado seguro social. La nueva norma reguló un caso adicional distinto de aquellos definidos en $\mathrm{N} 1^{\prime}$ y N2 dentro el sistema anterior.

En el año 1969 se produjo la sustitución de N2 (pensión de los empleados públicos) por N2'. Esta modificó los requisitos para obtener la pensión de jubila-

37 El 19 de febrero de 1945 se dictó la Ley 6a. Los artículos 14 y 17 crearon pensiones vitalicias de jubilación a favor de los trabajadores privados (con algunas restricciones) y públicos que hubiesen completado 20 años de servicios y 50 años de edad.

38 El artículo 260 del Decreto 2663 de 1950, mediante el cual se expidió el Código Sustantivo del Trabajo, dispuso el derecho a pensión mensual vitalicia de jubilación para ciertos trabajadores que hubiesen completado 20 años de servicios y 50 o 55 años de edad.

39 Con fundamento en disposiciones anteriores (Ley 90 de 1946) -que en nuestro criterio no alteran el universo de casos y soluciones de las pensiones de vejez y que por ello no son incluidas en la cadena de vigencia- el Gobierno nacional dictó el Decreto 3041 de 1966, de 19 de diciembre, por medio del cual se crea Instituto Colombiano de Seguros Sociales. Dentro de las condiciones para acceder al reconocimiento de la pensión de vejez propia de este, se estableció la existencia de un vínculo con la entidad (denominado aseguramiento), 60 o 55 años de edad, el pago de cotizaciones durante 500 semanas dentro de los 20 años anteriores al cumplimiento de esa edad, o durante 1000 semanas en cualquier tiempo (art. 11). Adicionalmente, se ordenó el reconocimiento pensional con un número de semanas inferior para aquellos nacidos antes de 1917, sin que en ningún caso la densidad de cotizaciones pudiera ser inferior a 250 semanas. 
ción, lo que dio lugar a un nuevo sistema S4. Por primera vez en la secuencia, se autorizó la aplicación de los mismos requisitos pensionales definidos en una norma derogada N2, pero se reserva dicha aplicación a supuestos fácticos específicos que ella misma definió. ${ }^{40}$

Posteriormente, sobrevino la derogación de N2' como consecuencia de la expedición de la Ley 33 de 1985, que en su artículo 1º (N2") estableció nuevas exigencias para el reconocimiento de la pensión de jubilación a algunos trabajadores del sector público, ${ }^{41}$ con lo cual surgió S5. El mismo artículo definió la aplicabilidad de las disposiciones anteriores en cuanto a la edad de jubilación (conforme a N2') para los trabajadores con 15 años de servicios al 29 de enero de $1985,{ }^{42}$ con lo cual nuevamente habilitó en esta secuencia la aplicación de normas anteriores.

Un nuevo sistema S6 surgió debido a la adición de una norma que estableció una nueva modalidad pensional con carácter general. El artículo 7o de la Ley 71 de 1988 creó la pensión de jubilación por aportes (N4), ${ }^{43}$ por medio de la cual se permitió, para efectos de obtener este derecho, la adición de tiempos de servicio al sector público y al sector privado. Así, el cambio de sistema surgió por la incorporación de un nuevo elemento (N4) al conjunto.

El último sistema de esta cadena (S7) surge por la modificación de N3, por N3', que derogó un aparte de la norma anterior que habilitaba a ciertas personas a obtener pensiones con un tiempo de cotización inferior. ${ }^{44}$

Conforme a la secuencia que se acaba de mostrar, se puede concluir que el primer orden jurídico objeto de análisis estuvo conformado por siete conjuntos consecutivos -sistemas-, el último de los cuales se integró, al menos en el tema de los requisitos para obtener las pensiones de vejez o jubilación, por los siguientes elementos:

$40 \quad$ El artículo 68 del Decreto 1848 de 1969, de 4 de diciembre, estableció el derecho a la pensión de jubilación para un subgrupo de trabajadores estatales denominado empleados oficiales, después de 20 años continuos o discontinuos de servicios y 50 o 55 años de edad, según se trate de mujeres u hombres respectivamente.

41 Conforme a esta, el derecho a la pensión de jubilación para el último subgrupo de trabajadores referido se hacía efectivo al cumplimiento de 20 años de servicios y 55 años de edad.

42 El parágrafo 2 de dicho artículo señala: "Para los empleados oficiales que a la fecha de la presente Ley hayan cumplido quince (15) años continuos o discontinuos de servicio, continuarán aplicándose las disposiciones sobre edad de jubilación que regían con anterioridad a la presente Ley".

43 La Ley 71 de 1988, de 19 de diciembre. Como se mencionó, el artículo 7 regula una nueva modalidad pensional denominada pensión de jubilación por aportes, aplicable a empleados del Estado y trabajadores con más de 55 y 60 años que hubiesen efectuado contribuciones durante 20 años o más.

44 El Decreto 758 de 1990, aprobatorio del Acuerdo 049 del mismo año, estableció en su artículo 12: "Requisitos de la pensión por vejez. Tendrán derecho a la pensión de vejez las personas que reúnan los siguientes requisitos: a) sesenta (60) o más años de edad si se es varón o cincuenta y cinco (55) o más años de edad, si se es mujer $y, b$ ) un mínimo de quinientas (500) semanas de cotización pagadas durante los últimos veinte (20) años anteriores al cumplimiento de las edades mínimas, o haber acreditado un número de un mil (1.000) semanas de cotización, sufragadas en cualquier tiempo". 
a. N0: regla de competencia presente en todos los sistemas desde el originario, y de la cual se derivan genéticamente todos los subconjuntos sucesivos.

b. N1': regula el caso genérico de las pensiones a cargo de empleadores privados.

c. N2": regula el caso genérico de las pensiones a cargo de empleadores públicos, mediante la derogación expresa de las normas anteriores, dentro de las que se encuentra N2'. Sin embargo, N2" "autorizó" la aplicación de esta bajo la expresión: "continuarán aplicándose las disposiciones sobre edad de jubilación que regian con anterioridad a la presente Ley”. Asimismo, N2' autorizó la aplicación de N2 al señalar que: "tendrán derecho a la pensión de jubilación al cumplir los veinte (20) años de servicios requeridos y cincuenta (50) años de edad, cualesquiera sea su sexo".

d. N3': regula el caso genérico de las pensiones a cargo del Instituto de Seguro Social a través de una operación de reparto.

e. N4: regula una pensión denominada pensión de jubilación por aportes que ampara el riesgo de la vejez

Conforme al aparato conceptual que se viene utilizando, es necesario precisar que a cada sistema jurídico correspondió un momento T. De esta manera, T0 corresponde a S0, y así sucesivamente.

La figura 1 da cuenta de la evolución de estos sistemas dentro de dicho orden.

Figura 1. Sistemas pensionales en Colombia. Evolución en forma de conjuntos en el marco de la Constitución Política de 1886
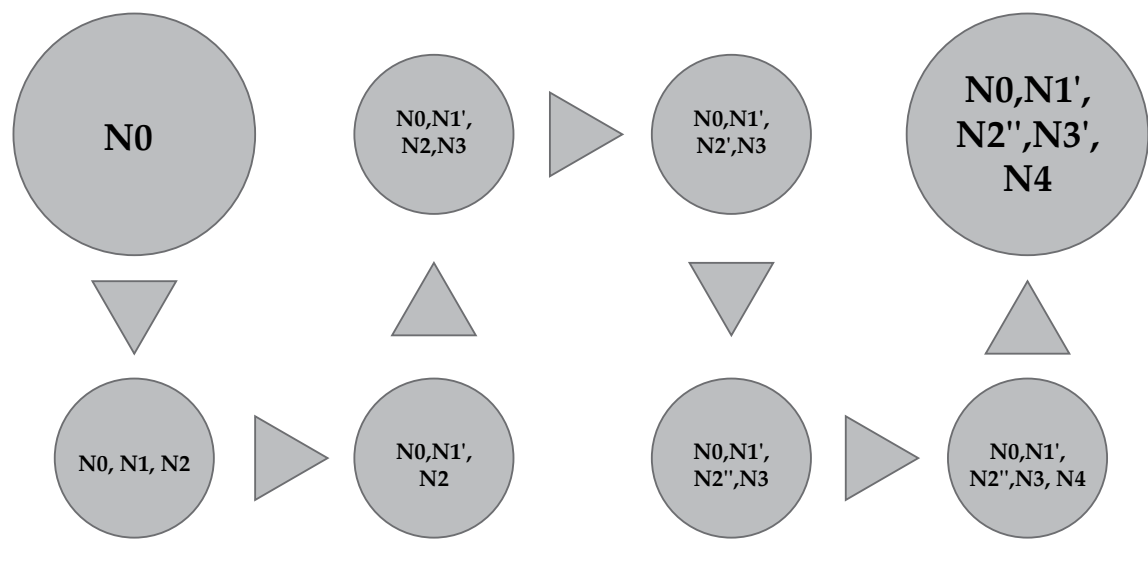

Fuente: elaboración propia.

\section{Orden jurídico actual: Constitución Política de 1991}

El orden jurídico colombiano actual (Oj1) nace en el año 1991 con la expedición de la actual Constitución Política, creada al margen de los procedimientos 
legales de reforma establecidos en la Carta anterior. ${ }^{45} \mathrm{El}$ tránsito de un orden jurídico a otro no implicó consecuencias inmediatas en el universo de casos de los que se ocupa la seguridad social, particularmente en lo que tiene que ver con las pensiones de vejez o jubilación.

De esta manera, al primer sistema jurídico de esta nueva secuencia ingresaron las normas pertenecientes al último sistema de la secuencia anterior, con un nuevo conjunto de normas independientes contenidas en la nueva Constitución Política $\left(\mathrm{N}^{\prime}\right)$ que reemplazó aquel contenido en la anterior. La incorporación de ese nuevo elemento nos lleva a predicar la existencia de un nuevo sistema dentro de una nueva secuencia.

El segundo momento de este orden surgió por la expedición de la Ley 100 en el año 1993, considerada el avance histórico más importante en materia de seguridad social en Colombia. En el caso de las pensiones de vejez, por ejemplo, se produjo la derogación de las normas anteriores que exigían diferentes requisitos (determinados por la existencia de un vínculo entre cada individuo y una institución determinada), y en su lugar se creó un sistema pensional universal que, en líneas generales, asignó la misma consecuencia jurídica a los mismos supuestos de hecho ${ }^{46}(\mathrm{~N} 5)$, sin perjuicio de algunas excepciones particulares que el mismo estatuto previó ${ }^{47}$ Asimismo, se incluyó expresamente la noción de "régimen de transición". El artículo 36 de este estatuto (N6) ordenó la aplicación de normas contenidas en el "régimen anterior" para los afiliados que cumplieran determinadas condiciones relacionadas con la edad o tiempo de servicios de las personas con expectativas de adquirir derechos pensionales, para la fecha en que la norma "entró en vigencia". ${ }^{48}$

Un nuevo sistema surgió en el año 2003 como consecuencia de la modificación de los requisitos para obtener la pensión (N5'). De esta manera, el legislador dispuso un incremento gradual del tiempo de servicios necesarios para obtener la pensión, además de una edad superior ${ }^{49}$ No obstante, las condiciones para

$45 \quad$ Véase el Decreto 927 de 1990.

46 El artículo 33 de la Ley 100 estableció que: “para tener derecho a la pensión de vejez, el afiliado deberá haber cumplido cincuenta y cinco (55) años de edad si es mujer, o sesenta (60) años de edad si es hombre, y haber cotizado un mínimo de mil (1.000) semanas en cualquier tiempo".

47 El Estatuto de la seguridad social excluyó expresamente de su aplicación: i) a los miembros de las Fuerzas Militares y de la Policía Nacional; ii) al personal civil vinculado a estas instituciones y regido por el Decreto Ley 1214 de 1990; iii) a los miembros no remunerados de las Corporaciones Públicas; iii) a los maestros afiliados a un fondo gremial denominado Fondo Nacional de Prestaciones Sociales del Magisterio; iv) a los trabajadores, que al momento de promulgación de la Ley estuviesen vinculados a empresas en concordato preventivo y obligatorio en el cual se hubiesen pactado sistemas o procedimientos especiales de protección de las pensiones, y mientras dicho concordato estuviese vigente; v) a los servidores públicos y pensionados de la Empresa Colombiana de Petróleos.

48 Esta norma señala que: “La edad para acceder a la pensión de vejez, el tiempo de servicio o el número de semanas cotizadas, y el monto de la pensión de vejez de las personas que al momento de entrar en vigencia el Sistema tengan treinta y cinco (35) o más años de edad si son mujeres o cuarenta (40) o más años de edad si son hombres, o quince (15) o más años de servicios cotizados, será la establecida en el régimen anterior al cual se encuentren afiliados. Las demás condiciones y requisitos aplicables a estas personas para acceder a la pensión de vejez se regirán por las disposiciones contenidas en la presente Ley".

49 Artículo 9. El artículo 33 de la Ley 100 de 1993 quedará así: "Artículo 33. Requisitos para obtener la Pensión de Vejez. Para tener el derecho a la Pensión de Vejez, el afiliado deberá reunir las siguientes condiciones: 1. 
obtener el derecho dentro del año siguiente fueron preservadas, pero a partir de 2005 se dispuso un incremento gradual del número de semanas exigidas, y desde 2014 el incremento de la edad.

En el año 2005 se expidió una norma que restringió la aplicabilidad de N5 (N7). Sin embargo, entre la fecha de su expedición y el año 2010 esta no produjo alteraciones en el universo de casos y soluciones de las pensiones pues dispuso la producción de sus efectos en dos etapas: hasta el año 2014 para los beneficiarios con más de 750 semanas cotizadas, y hasta 2010 para quienes no reunieran tal condición..$^{50}$

Así, el sistema jurídico que se estudia (con exclusión de los regímenes especiales mencionados) está conformado por el conjunto de normas independientes incluido en la Constitución vigente, mediante el cual se autoriza la promulgación y exclusión de normas a diferentes autoridades $\left(\mathrm{NO}^{\prime}\right)$, por la norma contenida en el estatuto de la seguridad social que estableció, desde 2014, el derecho a la pensión de vejez a las edades de 57 años para las mujeres y 62 para los hombres, junto con la exigencia de cotizaciones durante 1400 semanas (N5'), y, finalmente, por N7 que restringe la aplicabilidad de N6, la cual autorizaba la aplicación de las normas derogadas N1', N2", N3' y N4.

La figura 2 ilustra la secuencia, nuevamente, en términos de pertenencia.

\section{Figura 2. Sistemas pensionales en Colombia. Evolución en forma de conjuntos en el marco de la Constitución Política de 1991}

Fuente: elaboración propia.
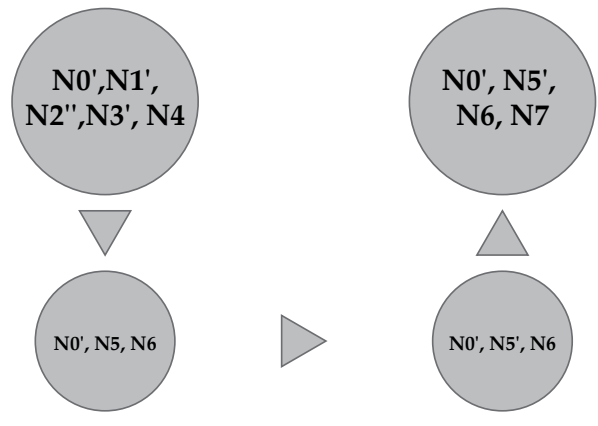

Haber cumplido cincuenta y cinco (55) años de edad si es mujer o sesenta (60) años si es hombre. A partir del $1^{\circ}$ de enero del año 2014 la edad se incrementará a cincuenta y siete (57) años de edad para la mujer, y sesenta y dos (62) años para el hombre. 2. Haber cotizado un mínimo de mil (1.000) semanas en cualquier tiempo [...] A partir del $1^{\circ}$ de enero del año 2005 el número de semanas se incrementará en 50 y a partir del $1^{\circ}$ de enero de 2006 se incrementará en 25 cada año hasta llegar a 1.300 semanas en el año 2015".

50 Acto Legislativo 01 de 2005: “Artículo 1o. Parágrafo transitorio 4o. El régimen de transición establecido en la Ley 100 de 1993 y demás normas que desarrollen dicho régimen, no podrá extenderse más allá del 31 de julio de 2010; excepto para los trabajadores que estando en dicho régimen, además, tengan cotizadas al menos 750 semanas o su equivalente en tiempo de servicios a la entrada en vigencia del presente Acto Legislativo, a los cuales se les mantendrá dicho régimen hasta el año 2014 [...] Los requisitos y beneficios pensionales para las personas cobijadas por este régimen serán los exigidos por el artículo 36 de la Ley 100 de 1993 y demás normas que desarrollen dicho régimen". 


\section{Casos problemáticos}

\section{Normas de transición: ambigüedad}

El análisis de las dinámicas anteriores resulta útil para identificar una primera dificultad relativa al concepto de normas de transición. Por ejemplo, en el año 1985 el legislador colombiano dispuso la adopción de una modificación de la norma que regulaba la pensión de jubilación para los empleados públicos con el fin de establecer una edad superior para causar ese derecho. Sin embargo, esa misma norma preservó la edad establecida en las reglas anteriores para un determinado grupo de personas, y expresó así una idea inicial de transición en los siguientes términos:

Los empleados oficiales en servicio activo que el día veintiséis (26) de diciembre de mil novecientos sesenta y ocho (1968), fecha de vigencia del Decreto Legislativo 3135 del año citado, hubieren cumplido diez y ocho (18) años de servicios, continua o discontinuamente, tendrán derecho a la pensión de jubilación al cumplir los veinte (20) años de servicios requeridos y cincuenta (50) años de edad, cualesquiera sea su sexo.

El análisis diacrónico efectuado es útil a fin de determinar qué otras normas permiten recoger un uso diferente de esa expresión (transición). El enunciado más significativo de esta clase (aunque no el único, pues uno similar ya había sido expresado normativamente en sistemas anteriores) es el artículo 36 del estatuto vigente de la seguridad social, que señala:

La edad para acceder a la pensión de vejez, el tiempo de servicio o el número de semanas cotizadas, y el monto de la pensión de vejez de las personas que al momento de entrar en vigencia el Sistema tengan treinta y cinco (35) o más años de edad si son mujeres o cuarenta (40) o más años de edad si son hombres, o quince (15) o más años de servicios cotizados, será la establecida en el régimen anterior al cual se encuentren afiliados.

Este contraste permite diferenciar dos tipos de normas de transición. De un lado, normas que extienden los efectos del sistema normativo y reiteran sus efectos restringiendo la aplicabilidad interna a través de un criterio personal. Por tanto, en estos casos, la nueva norma impone requisitos generales para obtener una pensión, pero crea una excepción para un grupo determinado de individuos que cumplen con una condición determinada, a quienes concede el derecho con los mismos requisitos de la norma anterior. En este caso, el efecto no se deriva de la anterior norma, sino de la nueva, que no modifica su situación.

De otro lado, surge otro tipo de normas de transición, que constituyen verdaderos criterios de aplicabilidad en sentido externo. Así estas, sin definir expresamente cómo debe ser regulada la instancia $c$ de un caso genérico $C$, establecen 
el deber de acudir a normas no pertenecientes (derogadas) con el fin de resolver las controversias respectivas.

En conclusión, las normas de transición pueden ser, al menos en el contexto del caso colombiano, normas que extienden la aplicabilidad interna de otras anteriores; o normas que actúan como criterios de aplicabilidad externo, mediante las cuales se habilita la aplicación de normas no pertenecientes.

\section{Indeterminación semántica}

De otro lado, en el apartado 3.2.2 se hizo referencia a la noción de aplicabilidad interna respecto de la cual Moreso y Navarro advierten una dificultad potencial relativa al proceso de subsunción de ciertos casos por efectos de la indeterminación. ${ }^{51}$

Asumiendo la idea de dimensión externa de aplicabilidad de las normas de transición, el caso colombiano muestra que el problema de indeterminación también puede afectar la aplicación de normas que sirven a este efecto. En el caso analizado, aun cuando está aceptado que durante los sistemas S1 y S2 del segundo orden jurídico el juez está obligado a aplicar normas anteriores, la expresión "disposiciones establecidas en el régimen anterior" puede estar referida, bien a normas aplicables, a normas pertenecientes, o a normas pertenecientes y aplicables.

Este tipo de problemas resulta en una dificultad ulterior para identificar las normas que se transmiten de un sistema a otro con restricciones en su aplicabilidad, debido a que todas las del sistema pertenecieron a algún régimen anterior. Esta particularidad implica, por efectos de la indeterminación en el lenguaje utilizado, un mayor grado de complejidad en la actividad de aplicación.

\section{Jerarquías y conflictos}

Las normas de transición habilitan la aplicación de normas no pertenecientes. Sin embargo, la aplicabilidad de estas también depende de otros criterios.

En el presente caso ha quedado ilustrado cómo en el sistema normativo 1 (S1) del orden jurídico 1 (O1) se introdujo un criterio de aplicabilidad externo (N6, norma de transición), que autorizó la aplicación de normas provenientes del orden jurídico anterior ( $\mathrm{N}^{\prime}, \mathrm{N} 2^{\prime \prime}, \mathrm{N} 3^{\prime}$ y N4'). Ahora, como se observa en la figura 2, en un momento posterior del orden surge un tercer conjunto por la incorporación de una norma constitucional N7, que redujo el ámbito de aplicación personal y temporal de N6, en cuanto autorizó el uso de la norma

51 Moreso y Navarro, "Applicability and effectiveness of legal Norms", op. cit. 
de transición solo para ciertos casos que cumplieran una condición determinada y hasta un momento definido. Debido a la introducción de este nuevo criterio, las instituciones solamente podían aplicar normas no pertenecientes (en virtud de esa norma de transición) hasta el año 2014.

Otro problema en el ordenamiento colombiano da cuenta de la existencia de conflictos entre normas de la misma jerarquía.

Por ejemplo, en el momento T4 de Oj0 (entre 1969 y 1985) la edad exigida a las mujeres para obtener una pensión de jubilación era de 50, mientras que la de los hombres era de 55 (N2'). Pese a ello, la norma estableció la posibilidad de reconocer pensiones a los hombres a la edad de 50 años (conforme establecía la norma derogada N2), siempre que cumplieran una condición determinada. Ahora, el sistema S5 surgió en 1985 como consecuencia de la modificación de ese requisito, e incrementó la edad de jubilación de las mujeres a 55 años. Sin embargo, en virtud de otra norma se habilitó la aplicación de "las disposiciones sobre edad de jubilación que regían con anterioridad a la presente Ley" para los empleados oficiales con 15 años de servicio a la fecha de promulgación. Posteriormente, esta norma fue objeto de derogación mediante la expedición de una nueva norma (N5), en un nuevo sistema (S2) de un nuevo orden (Oj1), la cual estableció nuevas condiciones pensionales, pero habilitó el uso de esta norma al cumplimiento de ciertas condiciones.

Así, existen dos reglas que autorizan la aplicabilidad de dos normas mutuamente excluyentes (N2" y N2'). Por un lado, N5 autoriza la aplicación de N2" (la cual establece la edad de pensión de los hombres a los 55), y, por el otro, N2" autoriza la aplicación de N2', la cual regula la edad de pensión de los hombres en 50 años. ¿Cuál es el criterio de aplicabilidad que debe ser seleccionado?

De esta manera, es importante advertir la posibilidad potencial de que se susciten conflictos entre criterios de aplicabilidad, que demandan el uso de criterios específicos para seleccionar las normas aplicables a cada caso concreto.

\section{Conclusiones}

El fenómeno de las normas de "transición" en los sistemas jurídicos de la seguridad social en Colombia se explica, desde la teoría del derecho de Alchourrón y Bulygin, en términos de pertenencia y aplicabilidad. En este sentido, el presente análisis resulta útil a fin de efectuar algunas breves aportaciones.

En primer lugar, el caso analizado constituye un nuevo ejemplo de aplicación de normas no pertenecientes, diferente de los que ya han sido recogidos por la bibliografía especializada. A la anterior conclusión se debe llegar necesariamente teniendo en cuenta que las normas "de transición" autorizan al aplica- 
dor del derecho a solucionar controversias utilizando normas que ya no hacen parte de los sistemas jurídicos por efectos de la derogación.

Correlativamente, el estudio de este tipo de sistemas provee un ejemplo adicional del fenómeno contrario, esto es, la pertenencia sin aplicabilidad, dado que la promulgación de normas de transición genera la imposibilidad de aplicar aquellas nuevas normas, debido a la existencia de un deber de acudir a normas excluidas de los sistemas.

Ahora, el análisis del caso mediante los instrumentos que provee la teoría del derecho resulta útil para advertir la existencia de algunas cuestiones no observadas por la literatura nacional, que se ha dedicado al análisis de la cuestión desde una perspectiva eminentemente dogmática.

Desde el punto de vista de la seguridad social, la idea de la transición sugiere el cambio de un sistema normativo por otro. Sin embargo, el presente trabajo ha sido útil para identificar una ambigüedad en el uso de la expresión. Así, la transición de un sistema normativo a otro ha sido adoptada en dos formas: i) mediante la expedición de normas que reiteran parcialmente el contenido de aquellas derogadas, pero restringen su ámbito de aplicabilidad interno, como en el caso del artículo $1^{\circ}$ de la Ley 33 de 1985, que mantiene el derecho al reconocimiento de la pensión establecida en normas derogadas si y solo si se cumplen ciertos requisitos; ${ }^{52} \mathrm{o}$ ii) mediante la adopción de normas que actúan como criterios de aplicabilidad externa, como en el caso del artículo 36 de la Ley 100 de 1993, que deroga las disposiciones anteriores, pero que autoriza la aplicación siempre condicionada al cumplimiento de ciertos requisitos. De esta forma, dicho trasunto ocurre, i) bien a través de la extensión de la aplicabilidad interna, o ii) mediante la promulgación de normas que actúan como criterios de aplicabilidad externa.

La ubicación del fenómeno dentro de la teoría de los sistemas jurídicos permite, de un lado, entender la funcionalidad de los conceptos de aplicabilidad interna y externa, especialmente la relación de dependencia que sobre el particular plantean algunos especialistas, $\mathrm{y}$, de otro, advertir una ambigüedad referenciada en el uso de la terminología.

Finalmente, se ha podido verificar que la aplicabilidad de las normas no solo depende de criterios externos o internos, pues estos pueden estar limitados por otras normas del sistema. Por tanto, dentro de este tipo de dinámicas existe la posibilidad potencial de que se susciten conflictos entre criterios de aplicabilidad, para lo cual existe la necesidad de acudir a ciertos criterios de los que se puedan valer las instituciones para seleccionar las normas aptas a cada caso concreto.

52 Supra, apartado II, numeral 2. 


\section{Bibliografía}

Alchourrón, Carlos y Eugenio Bulygin, Normative Systems, Springer, Wien, 1971.

Alchourrón, Carlos y Eugenio Bulygin "Sobre el concepto de orden jurídico", Crítica 23 (1976), pp. 3-21.

Bulygin, Eugenio, "Time and Validity”, en Análisis lógico y derecho, Centro de Estudios Constitucionales, Madrid, 1991, pp. 195-215.

Bulygin, Eugenio, "Algunas consideraciones sobre los sistemas jurídicos", en Doxa 9, (1991b), pp. 257-279. https://doi.org/10.14198/DOXA1991.9.13

Bulygin, Eugenio y Daniel Mendonça, Normas y sistemas normativos, Madrid, Marcial Pons, 2005.

Caracciolo, Ricardo, La noción de sistema en la teoría del derecho, México D.F., Fontamara, 1994.

Caracciolo, Ricardo, El sistema jurídico: problemas actuales, Madrid, Centro de Estudios Constitucionales, 1988.

Ferrer, Jordi, Las normas de competencia: un aspecto de la dinámica jurídica, Madrid, Centro de Estudios Políticos y Constitucionales, 2000.

Ferrer, Jordi y Jorge L. Rodríguez, Jerarquías normativas y dinámicas de los sistemas jurídicos, Madrid, Marcial Pons, 2011.

Moreso, José Juan y Pablo Navarro, Orden jurídico y sistema jurídico. Una investigación sobre la identidad y dinámica de los sistemas jurídicos, Madrid, Centro de Estudios Constitucionales, 1993.

Moreso, José Juan y Pablo Navarro, “Applicability and effectiveness of legal Norms", en Law and Philosophy 16 (2005), pp. 201-219, DOI: https://doi. org/10.1023/A:1005884330974

Moreso, José Juan y Pablo Navarro, "The reception of norms, and open legal systems", en S. Paulson y B. Litschewsky (eds.), Normativity and Norms: Critical perspectives on Kelsenian Themes, Oxford, Oxford University Press, 1999.

Moreso, José Juan y Pablo Navarro, "Some remarks on the notions of Legal Order and Legal System", en Normas jurídicas y estructura del Derecho, México, Fontamara, 2002, pp. 13-34.

Moreso, José Juan y Josep María Vilajosana, Introducción a la teoría del derecho, Madrid, Marcial Pons, 2004.

Raz, Joseph, The concept of a legal system. An introduction to the theory of a Legal System, Oxford, Clarendon, 1980. 
Rodríguez, Jorge Luis, Lógica de los sistemas jurídicos, Madrid, Centro de Estudios Políticos y Constitucionales, 2002.

Vilajosana, Josep M., El significado político del Derecho, México, Marcial Pons, 1997.

Vilajosana, Josep M., "Dinámica de sistemas y persistencia de normas jurídicas", Doxa 21 (I) (1998), pp. 49-64. DOI: https://doi.org/10.14198/ DOXA1998.21.1.03

\section{Fuentes jurídicas nacionales}

Código Sustantivo del Trabajo.

Congreso de Colombia, Ley 6 ${ }^{\mathrm{a}}, 5$ de febrero de 1945, DO: 25790.

Congreso de Colombia, 26 de diciembre de 1946, Ley 90, DO: 26322.

Congreso de Colombia, 29 de enero de 1985, Ley 33, DO: 36856.

Congreso de Colombia, 19 de diciembre de 1988, Ley 71, DO: 38624.

Congreso de Colombia, 23 de diciembre de 1993, Ley 100, DO: 41148.

Congreso de Colombia, 29 de enero de 2003, Ley 797, DO: 45079.

Congreso de Colombia, 22 de julio de 2005, Acto Legislativo 01, DO: 45980.

Constitución Política de Colombia, 5 de agosto de 1886.

Constitución Política de Colombia, 6 de julio de 1991.

Presidencia de la República, Ministerio del Trabajo, 19 de diciembre de 1966, Decreto 3041, DO: 32126.

Presidencia de la República, Ministerio del Trabajo, 4 de noviembre de 1969, Decreto 1848, DO: 32937.

Presidencia de la República, Ministerio del Trabajo, 11 de abril de 1990, Decreto 758, DO: 39303. 